\title{
EDITORIAL
}

\section{Vascular Complications in Diabetes}

\author{
Raquel Soares*
}

Type 2 Diabetes Mellitus (T2DM) is a chronic disease characterized by impaired fasting and post-prandial glucose tolerance, which is associated with insulin resistance and hyperinsulinemia among other secondary metabolic changes [1]. Despite the increasing knowledge on this complex disease, its incidence and prevalence rates continue to increase all over the world [2]. This has been attributed to the rising number of people adopting the western life-style. Indeed, excessive energy intake and low physical activity, accompanied by a long-term, continuous and psychological stress that characterizes the society of the XXI century, are considered causative factors. In addition, low quality food and disruption of circadian rhythms, such as shift work and other sleeping time disturbances, further contribute to the increase in diabetes and other clustered metabolic disorders [3].

The clinical manifestations of diabetes comprise profound alterations in the function of many organs, resulting often in nephropathy, retinopathy, accelerated atherosclerosis, neuropathy and impaired wound healing, many of these involving vascular complications. Vascular disturbances are, thus, features of diabetic patients, both affecting large and small vessels. These micro and macrovascular complications greatly contribute to the high morbidity and mortality rates of diabetes [1, 4]. Angiogenesis and its counterpart, vasculogenesis are two processes deeply implicated in diabetes. Nevertheless, this association is somehow paradoxal. Enhanced neovascularization occurs in diabetic kidney, retina and associated with the growth of vasa vasorum within atheroma plaques, often leading to intraplaque hemorrhage and plaque disruption, which ultimately contributes to elevated cardiovascular risk of diabetic patients. Conversely, neovascular impairment is also observed in diabetes, namely contributing to skin wound injury, and ischemic episodes that may result in decreased myocardial perfusion [4]. This vascular paradox, defined by the simultaneous presence of stimulating and inhibitory neovascular processes in diabetes, has been attributed to organ-specific features that interfere with endothelial cell proliferation (angiogenesis) and endothelial progenitor cells differentation (vasculogenesis). The molecular mechanisms underlying this angio-vasculogenic paradox are highlighted in the first two papers of this issue. Moreover, a careful evaluation of the putative and currently used therapeutic approaches is also provided in these articles.

Recent advances on peptide hormones, such as ghrelin, an orexigenic hormone, and related peptides emphasize their involvement in diabetic pathogenesis [5]. In agreement, ghrelin mediates glucose and lipid metabolism, being further implicated in insulin secretion as well [5]. Controversial evidence has been gathered regarding the role of ghrelin as a pro- or antiangiogenic factor. These findings are, therefore, summoned by Rocha-Sousa et al. in the present issue, given their novelty and the potential effect of this hormone in the development of diabetic retinopathy.

A feature of proliferative diabetic retinopathy is the presence of excessive vascularization in the retina. Being the major cause of blindness in the active population, and hence, one of the main morbidity conditions of diabetes, retinopathy is a relevant debilitating condition. Several lines of research have been recently undertaken, in order to elucidate the vascular abnormalites of the retina and to design new therapies directed against angiogenesis and vasculogenesis within the diabetic eye. The inherent therapeutic advances already in clinical use and their putative drawbacks are also described by Falcão and collaborators in the current issue. Finally, vascular impairment in skin wound healing strongly correlates with chronic inflammatory and neuropathy-associated peptides. Diabetic foot ulcer development and progression is an expensive condition, strongly demanding a thorough clinical management. Therefore, understanding the crosstalk between neuropeptides, inflammatory mediators and vascular abnormalities is of paramount importance. This interplay is highlighted by Tellechea et al. Although the awareness regarding the vascular paradox in diabetes is currently increasing, the underlying molecular events are still a matter of debate. Identifying these will enable the development of therapeutic strategies that are in need for the increasing burden of T2DM, which is already reaching epidemic proportions worldwide.

\section{REFERENCES}

[1] Stratton, I. M.; Adler, A. I.; Neil, H. A.; Matthews, D. R.; Manley, S. E.; Cull, C. A.; Hadden, D.; Turner, R. C.; Holman, R. R. Association of glycaemia with macrovascular and microvascular complications of type 2 diabetes (UKPDS 35): prospective observational study. Br. Med. J., 2000, $321,405-12$

[2] Wild, S.; Roglic, G.; Green, A.; Sicree, R.; King, H. Global prevalence of diabetes: estimates for the year 2000 and projections for 2030. Diabetes Care, 2004, 27, 1047-53. 
[3] Azevedo, A.; Santos, A.; Ribeiro, L.; Azevedo, I. In The Metabolic Syndrome: Oxidative stress, inflammation and angiogenesis in metabolic syndrome. Soares, R.; Costa, C.; Eds.; Springer-Verlag: The Netherlands, 2009, pp. 1-19.

[4] Waltenberger, J. New Horizons in Diabetes Therapy: The Angiogenesis Paradox in Diabetes: Description of the Problem and Presentation of a Unifying Hypothesis. Immunol. Endocr. Metab Agents - Med Chem., 2007, 7, 87-93.

[5] Kojima, M.; Hosoda, H.; Date, Y.; Nakazato, M.; Matsuo, H.; Kangawa, K. Ghrelin is a growth-hormone-releasing acylated peptide from stomach Nature, 1999, 402, 656-60.

Raquel Soares

Department of Biochemistry (U38),

Faculty of Medicine, University of Porto,

Al. Prof. Hernâni Monteiro,

4200-319 Porto,

Portugal,

Tel/Fax: 351225513624 ,

E-mail: raqsoa@med.up.pt

(C) Raquel Soares; Licensee Bentham Open.

This is an open access article licensed under the terms of the Creative Commons Attribution Non-Commercial License (http: //creativecommons.org/licenses/by$\mathrm{nc} / 3.0 /$ ) which permits unrestricted, non-commercial use, distribution and reproduction in any medium, provided the work is properly cited. 